\title{
Strong angular dependence of resonant states in 2D dielectric cylinder rings
}

Cite as: J. Appl. Phys. 124, 174903 (2018); https://doi.org/10.1063/1.5047059

Submitted: 04 July 2018 . Accepted: 19 October 2018 . Published Online: 05 November 2018

Ángel Andueza, Jesús Pérez-Conde (iD), and Joaquín Sevilla (iD)
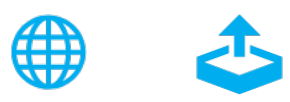

\section{ARTICLES YOU MAY BE INTERESTED IN}

Theory of optical forces on small particles by multiple plane waves

Journal of Applied Physics 124, 173102 (2018); https://doi.org/10.1063/1.5046154

Shielding effectiveness and bandgaps of interpenetrating phase composites based on the Schwarz Primitive surface

Journal of Applied Physics 124, 175102 (2018); https://doi.org/10.1063/1.5046105

Hierarchically structured metamaterials with simultaneously negative mass density and Young's modulus by using dynamic homogenization

Journal of Applied Physics 124, 175103 (2018); https://doi.org/10.1063/1.5050207

\section{Journal of Applied Physics Polymer-Grafted Nanoparticles}




\title{
Strong angular dependence of resonant states in 2D dielectric cylinder rings
}

\author{
Ángel Andueza, ${ }^{1,2, a)}$ Jesús Pérez-Conde ${ }^{3}$ and Joaquín Sevilla ${ }^{1,2}$ \\ ${ }^{1}$ Dpto. Ing. Eléctrica, Electrónica y de Comunicación, Universidad Pública de Navarra, 31006 Pamplona, \\ Spain \\ ${ }^{2}$ Smart Cities Institute, Universidad Pública de Navarra, 31006 Pamplona, Spain \\ ${ }^{3}$ Dpto. de Física, Universidad Pública de Navarra, 31006 Pamplona, Spain
}

(Received 4 July 2018; accepted 19 October 2018; published online 5 November 2018)

\begin{abstract}
We investigate collective resonators made of dielectric cylinders assembled as two-dimensional regular octagons and decagons. These structures exhibit collective resonance states that change their intensity with the incident radiation angle. While most parts of the spectra present small or even null variation, one of these resonances presents high-sensitivity to the incidence angle. This strong variation is well characterized in terms of the electric field intensity distribution of a resonant state where all the cylinders show the lowest order Mie resonance and the neighbors alternate their polarities. The collective state is optimally excited when radiation impinges on a vertex of the polygonal arrangement of cylinders, while the response decreases to its minimum when the incident field hits an edge (two cylinders at the same time). The resonant state and its high dependence on the excitation incidence angle have been found in both octagonal and decagonal configurations for different dielectric permittivity values. In addition, the scalability of Maxwell equations warranties the same behavior if the whole system is downscaled to terahertz or optic frequencies. The study was performed by finite integration time domain calculations of scattering and transmission for different incidence angles. Experimental measures in the microwave range were taken from photonic molecule prototypes made of centimeter-scale glass cylinders $(\varepsilon=4.5)$. We find an overall excellent agreement between measurements and simulations. We propose that photonic molecules made of polygonal rings of dielectric cylinders are an ideal structure to build angle sensors using the strongly varying state that they present. Published by AIP Publishing. https://doi.org/10.1063/1.5047059
\end{abstract}

\section{INTRODUCTION}

Photonic crystals (PhCs) and, later, metamaterials marked a breakthrough in light control and manipulation. Photonic crystals (PhCs) are created with different structures: regular, quasicrystalline, ${ }^{3,4}$ or disordered ${ }^{5,6}$ with distances of the same order of the light wavelength. Smaller structures made of resonators and arrays of resonators (photonic molecules) ${ }^{7}$ have also been extensively studied over the last decade producing novel designs such as Fabry-Pérot resonators, ${ }^{8}$ spheres, ${ }^{9}$ rings, ${ }^{10}$ disk, ${ }^{11,12}$ defect cavities in photonic crystal, ${ }^{13}$ coupled resonator optical waveguides (CROWs), ${ }^{14}$ and rods. ${ }^{7,15,16}$ These structures have been proved in a number of applications such as enhanced semiconductor microlasers, ${ }^{11,17}$ cavity optomechanics, ${ }^{10,18}$ cavity quantum electrodynamics experiments, ${ }^{19}$ classical and quantum information processing, ${ }^{20}$ and optical devices as switches and filters. ${ }^{21-23}$ Also, resonator systems have been proposed as sensor devices for refractive index, ${ }^{15}$ stress, ${ }^{24}$ or rotation, ${ }^{25-27}$ among other measurement variables.

This work explores the electromagnetic properties and applications of collective resonators (CRs) based on dielectric rods with octagonal and decagonal symmetries, paying special attention to their rotation angle with respect to the incident radiation direction. In order to complete the understanding of the basic behavior of these systems, we have numerically studied their scattering response and electric field distribution.

\footnotetext{
a) Author to whom correspondence should be addressed:

angel.andueza@unavarra.es
}

Octagonal and decagonal clusters of glass cylinders were built (see Fig. 1), and their microwave transmission spectra were measured for different incidence angles. Two modes are clearly observed in the transmission response as the structure is rotated. One of these modes is sensitive to angle rotation, while the other mode is almost independent of it. These modes could be used as a differential sensor where the angular position of the structure would be determined by the difference of the transmission intensity between them.

\section{EXPERIMENTAL}

\section{A. Cylinder ring resonators analyzed}

The selected resonator structures are rings of cylinders with $n$-fold rotational symmetries that naturally appear in 2D photonic Quasicrystals (QCs) and that have been extensively studied. $^{4,16,28,29}$ Studies about light localization in QC with octagonal, decagonal, and dodecagonal high-symmetry local centers find that, in general, high-symmetry local clusters are responsible for discrete states inside complete photonic bandgaps in quasiperiodic structures. ${ }^{16}$ The high-symmetry centers of cylinders inside the QCs can be considered as clusters which present a set of states arising from the isolated Mie resonances of the individual cylinders. ${ }^{4}$ The light localization regime in octagonal and decagonal clusters is determined by the geometrical and dielectric properties of these clusters of scatterers, but it is independent of the local symmetry surrounding them. ${ }^{16}$ This means that we can regard quasiperiodic $\mathrm{PhC}$ as a disordered media, responsible for a 



FIG. 1. Structure of the collective resonators studied in this paper. Resonators are made of glass dielectric cylinders arranged as a ring in octagonal [(a) and (c)] and decagonal [(b) and (d)] symmetries. Axis labeling and definition of the design parameters in (a) and (b). Pictures of the actual prototypes built for measurement are shown in (c) and (d).

quite flat bandgap, and cylinder ring resonators, responsible for localized photonic states (some of them inside the gap). To our knowledge, an analysis of these high-symmetry local centers on their own, outside from QC structures, has not been previously performed and is the aim of this paper.

In order to study the above mentioned resonator structures, we built several samples to be probed in the range of microwaves. Two rings with decagonal and octagonal symmetries were fabricated using soda-lime glass cylinders (see Fig. 1) of radius $r=3 \mathrm{~mm}$ (with a dispersion of less than $3 \%$ ) and length $\mathrm{L}=250 \mathrm{~mm}$. Cylinders were held parallel to each other and fixed between two wooden pieces where their positions were previously carved with a numerical control drill. The radial distance in both cases was $a=12 \mathrm{~mm}$. The aspect ratio (AR) of the structure, defined as $A R=L / 2$ r, was 33. This value was chosen to assure a negligible influence of the finitude in the $\mathrm{x}$-direction on the measurements, which is attained for AR > 10 (as stated in basic texts of electromagnetism like Bohren and Huffman). ${ }^{30,31}$

We have performed the measurements and calculations in the interval between the two extreme cases of azimuth angle: at $0^{\circ}$ and $18^{\circ}\left(22.5^{\circ}\right)$, as the situation repeats once the angle reaches these values due to rotational symmetry.

\section{B. Experimental setup}

Transmission spectra of the samples were measured using a vector network analyzer (VNA) HP 8722ES spliced to rectangular horn antennas $79 \times 46 \times 129 \mathrm{~mm}$ (Narda Model 640), aligned, and separated approximately $350 \mathrm{~mm}$ from each other. The sample is placed over a rotating platform in the space between the antennas, with the cylinders perpendicular to the incidence direction. ${ }^{15,31}$ The rotating platform allows one to change the incidence angle of the radiation in the sample (rotation in the $y z$ plane).
The measurement frequency ranges from $8 \mathrm{GHz}$ to $12 \mathrm{GHz}$. A differential measurement technique was used to register the transmission spectra. First, the transmission spectrum without the sample was recorded on the network analyzer. Next, the transmission spectrum of the structure was measured. The analyzer automatically provided the difference between the spectra with and without the sample. A smoothing algorithm Savitzky-Golay from VNA (span for the moving average of 6 samples) was used to reduce the ripple noise of the measurements. The number of samples in the experimental transmission measurements is 1601 , which limits the maximum resolution of the measurements to $\Delta \sim 2.5 \mathrm{MHz}$. An outline of the experimental setup can be seen in Fig. 2. ${ }^{15,31}$

\section{Calculation procedure}

Numerical calculations were carried out with CST MICROWAVE STUDIO, a commercial code based on the Finite Integration time-domain Method (FIM). ${ }^{32}$ This program is an electromagnetic field simulation software package, especially suited for analysis and design in the high-frequency range.

In this work, we employed two different solver tools available in the CST MW Studio. A frequency solver with a tetrahedral adaptive mesh refinement was used to calculate the scattering cross section (SCS) of clusters using infinite length cylinders $(\mathrm{L}=\infty)$. A transient solver with a hexahedral adaptive mesh refinement was employed for the calculations of transmission spectra of finite CRs (cylinders with a finite length). Following the specifications of the actual antennas used in the experimental setup, two horn antennas were modeled in the CST MW Studio. Besides, two waveguide ports (WR-90) of size $22.86 \times 10.16 \mathrm{~mm}$ were coupled to the simulated antennas for the calculations of finite samples measured. Horn antennas were simulated exciting the structure with an electric field polarized along the $\mathrm{x}$-axis (TM polarization). In all cases (measured and calculated), we keep the axis definition as shown in Fig. 2, where $\mathrm{z}$ is the direction of radiation propagation and $\mathrm{x}$ is the cylinder length direction.



FIG. 2. Outline of the experimental setup. The sample is placed at the center of a holder that can rotate. The cylinders are perpendicular to the depicted plane (in the $x$ direction), while radiation is emitted and detected perpendicularly ( $\mathrm{z}$ direction) by horn antennas connected to a vector network analyzer (VNA). The only moving part is the sample holder that rotates allowing one to change the sample angle with respect to the radiation line of emission and detection. 


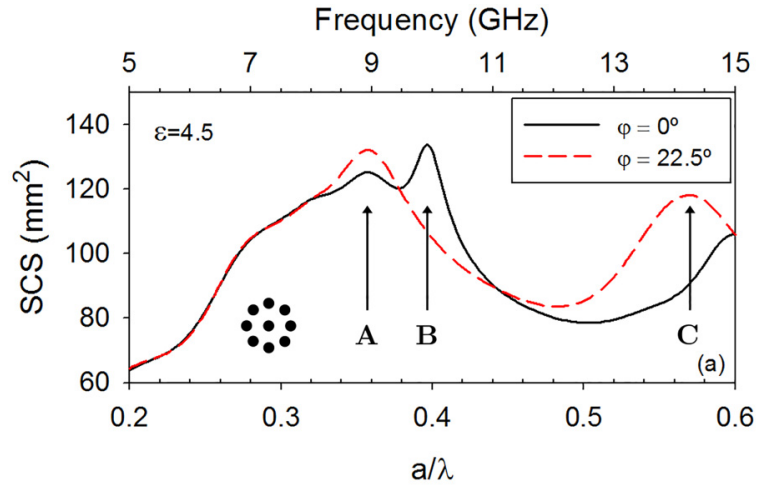

Frequency $(\mathrm{GHz})$



FIG. 3. Scattering Cross Section (SCS) as a function of the normalized frequency for the (a) octagonal and (b) decagonal CR at normal incidence (a solid black line) and at maximum angle given by the rotational discrete symmetry of the structure (a red dashed line). The parameters employed by the calculations were $\varepsilon=4.5, \mathrm{a}=12 \mathrm{~mm}$, and $\mathrm{r}=0.25 \mathrm{a}$.

\section{RESULTS AND DISCUSSION}

\section{A. Scattering cross section (SCS)}

We first calculate the scattering cross section (SCS) of the two structures under study, octagonal and decagonal rings. The results are shown in Fig. 3. Frequency is presented as the normalized parameter $(\mathrm{a} / \lambda)$ as well as in the actual
TABLE I. Summary of the frequency position of the peaks found in Fig. 3.

\begin{tabular}{lccrr}
\hline \hline & & A & B & \multicolumn{1}{c}{ C } \\
\hline Octagonal & $\mathrm{a} / \lambda$ & 0.36 & 0.40 & 0.57 \\
& $\mathrm{f}(\mathrm{GHz})$ & 8.93 & 9.91 & 14.23 \\
Decagonal & $\mathrm{a} / \lambda$ & 0.42 & 0.46 & 0.58 \\
& $\mathrm{f}(\mathrm{GHz})$ & 10.44 & 11.50 & 14.47 \\
\hline
\end{tabular}

frequency units $(\mathrm{GHz})$. Two lines are plotted for each structure representing the SCS when the orientation of the structures with respect to the incident radiation is in the two extreme angular values (see Fig. 1).

Both CRs present scattering spectra with very similar behavior. For low frequencies, the SCS of each CR is identical for the two extreme orientations. At higher values, 3 peaks can be identified (labeled A, B, and C in the figures), whose value changes for the two angles represented. When the structure is rotated, the first broad peak (A) frequency remains unaltered, whereas the intensity is slightly modified. Meanwhile, the second peak (B) disappears totally. The third peak $(\mathrm{C})$ is not present at $0^{\circ}$ and grows for the increased angle. The position of peaks $\mathrm{A}, \mathrm{B}$, and $\mathrm{C}$ for the two structures is summarized in Table I.

\section{B. Electric field distributions}

In order to obtain a more detailed view of the resonance states of the structures, we computed the spatial distribution of electric field for peaks A and B. In the calculation, polarized radiation in the $\mathrm{x}$-direction with unit amplitude propagating in the z-direction was taken. The electric field distributions of peaks A and B in plane $y z$ for both octagonal and decagonal clusters are depicted in Fig. 4.

In both cases, peak B undergoes a critical reduction of the electric field in comparison with the structure at $0^{\circ}$. The influence of rotation is weaker for peak $\mathrm{A}$, where the $0^{\circ}$
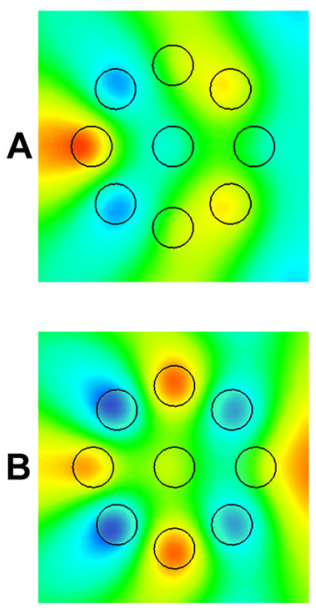

$22.5^{\circ}$


(a) $0^{\circ}$
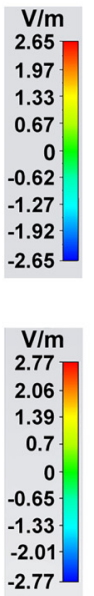

$\mathrm{V} / \mathrm{m}$
2.77
2.06
1.39
0.7
0
-0.65
-1.33
-2.01
-2.77

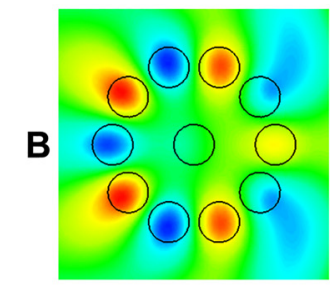

$18^{\circ}$
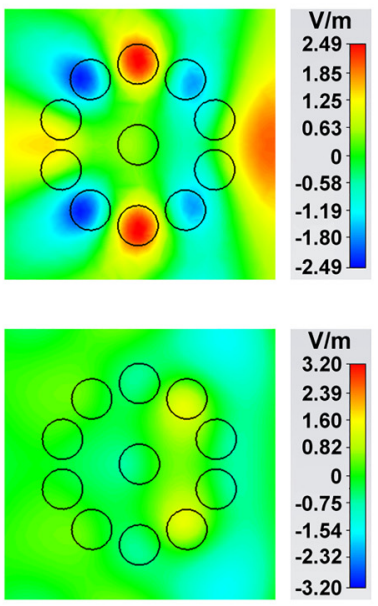

(b)

FIG. 4. Distribution of the electric-field in the $y z$ plane corresponding to peaks A and B (defined in Figure 3) for (a) the octagonal CR with an incidence angle of $\varphi=0^{\circ}$ and $\varphi=22.5^{\circ}$ and (b) decagonal CR with an incidence angle of $\varphi=0^{\circ}$ and $\varphi=18^{\circ}$. The red and blue colors indicate the electric field intensity and polarity, and green color indicates null value for the electric field. It can be seen that peak B at $0^{\circ}$ presents field maxima in each cylinder, alternating polarities. 
magnitude of the electric field is similar in the two angles presented. Especially remarkable is the mismatch in the field distribution of peak B between the two angular positions. The influence of rotation does not seem to affect so critically to peak A, where the magnitude hot spots of the electric field are similar in the two angles presented.

These maximum peaks in the SCS function correspond to localized states, particularly field patterns confined in the ring of cylinders. ${ }^{16}$ These collective states result from the combination of individual Mie resonances in various cylinders at a local scale and the rotational symmetry imposed by the geometry of the rings. The same electric field intensity distributions have been previously found in structures which contain decagonal and octagonal clusters of cylinders such as quasicrystals and Penrose's tilling. ${ }^{4,16}$ What we observe here is a great difference in the excitation of these patterns depending on the radiation incidence angle. Peak B corresponds to a structure formed by field maxima alternating positive and negative polarization with respect to the $\mathrm{x}$-direction in each of the cylinders. This field distribution is excited when the radiation wavefront hits a single cylinder when impinging on the structure $\left(0^{\circ}\right.$ in our reference frame) and it is symmetric with respect to the z-axis. However, if the radiation finds two cylinders at the same time when the ring is rotated, then it is impossible to drive them with alternate polarities and it is symmetric with respect to the $\mathrm{z}$-axis, making impossible the excitation of the mode. On the contrary, peak A corresponds to a field distribution where field maxima are shared by two cylinders if the octagon (decagon) is rotated $22.5^{\circ}$ $\left(18^{\circ}\right)$ and it is also symmetric with the propagation axis, which allows very similar patterns of excitation when the radiation hits in a single cylinder at $0^{\circ}$ as can be seen in Figs. 4(a) and 4(b).

\section{Transmission measurement and simulation}

We measured the transmission of physical prototypes of the structures under study. The same geometrical configuration of the measurement (including the antennas) was also modeled in the simulator to obtain the corresponding transmission values. ${ }^{31}$ It is important to note different calculation procedures in the case of the SCS (where plane wave excites an infinite structure) and in the case of transmission measurements (where a finite sample is illuminated by finite antennas). The results of these measurements and calculations are presented in Fig. 5. The figure shows threedimensional representations where transmission spectra (transmittance versus normalized frequency) are piled at a third axis for the different angles of the incident radiation. The transmission value is also depicted in a color scale to make it easier to follow the variations.


FIG. 5. Color map plots of calculated (left) and measured (right) transmission spectra for a decagonal CR as a function of normalized frequency (a/ $\lambda$ ) and angle $(\varphi)$. Upper figures [(a) and (b) are for the octagonal ring and lower figures [(c) and (d)] are for the decagonal ring. Arrows and black dashed lines show the frequency position and transmission variation of peaks $\mathrm{A}$ and $\mathrm{B}$ with respect to the angle. 




FIG. 6. Evolution of normalized differential transmission parameter $\Delta \mathrm{T} / \Delta \mathrm{T}_{0}$ in the octagonal $\mathrm{CR}$ as a function of the azimuth angle. Black circles correspond to calculations and white circles to measurements. The lines correspond to linear fitting of each set of points.

As it can be seen, the agreement between calculation and measurements is remarkable. The general outline of the plots is similar, with good coincidence in the transmittance values. Both peaks are found at the same frequency and present the same evolution with angle (one diminishing and one not), which is an important feature. However, the experimental measurement is much noisier. For example, in the 0.32 to $0.36 \mathrm{a} / \lambda$ interval of the upper curves, the calculation presents a smooth curve with only one local minimum, while the noisier experimental curve presents four minima. There are a number of sources of error in the experiment that may account for these discrepancies: the fabrication tolerances of the sample, its positioning (linear and angular), and the antennas alignment. Particularly important is the fact that the sample is much smaller than the illuminated area of the antennas and, as the measurements were not made in an anechoic chamber, rebounds and undesired interference may be taking place.

Besides, the agreement between experimental and calculated, transmission data are in accordance with SCS data previously analyzed, not only in the profile of transmission spectra but also in the position of peaks A and B. These peaks behave as expected from the SCS, while A remains almost unchanged, B decreases significantly its value as the angle varies.

\section{Potential use as an angle sensor}

The existence of two states that show transmission and scattering properties, so different in terms of the rotation angle $\varphi$, is an interesting feature for angle sensors design. A differential sensor can be made based on the state which is strongly dependent on the angle (B) and another state invariant under angle rotations, where the value of the angle would be determined by the difference of the transmission intensity value between the two states. ${ }^{15}$ In general, differential operation in sensors provides multiple advantages such as systematic error cancellation, cross-sensitivities, and noise reduction.

As a proof of concept of this idea, we have drawn a calibration curve for the octagonal structure based on the data presented in Fig. 5. We define $\Delta \mathrm{T}$ as a differential transmission value between frequency values of the resonances shown by the structures, $\Delta T=\left|T_{B}-T_{A}\right|$, where $T_{B}$ corresponds to transmission at the frequency of resonance dependent on the azimuth angle and $\mathrm{T}_{\mathrm{A}}$ corresponds to transmission at the frequency of resonance independent of the azimuth angle. Next, we compute the values of $\Delta \mathrm{T}$ using the calculated and measured transmission spectra of the samples (Fig. 6). Figure 6 shows the result for the octagonal structure. These values of $\Delta \mathrm{T}$ are plotted, normalized to $\Delta \mathrm{T}$ at angle $\varphi=0^{\circ}\left(\Delta \mathrm{T}_{0}\right)$, in Fig. 6, where we observe that the agreement between the measurement and calculated data is good. The calibration curve obtained presents an S-shape, typical in many instrumentation systems, but it can be linearly approximated without much error.

These results are more general than the detailed cases studied here. Although the presented structures have been tested in the microwave range, the scaling properties of Maxwell equations allow a direct extrapolation to other electromagnetic regimes. It is also important to use materials with the same dielectric permittivity in the desired regime. The permittivity value in this work was $\varepsilon=4.5$ of the glass rods used in the experiments; however, the same states are also found for higher values of the dielectric permittivity. For example, results for $\varepsilon=13$ are shown in Fig. 7, where a much higher Q-factor is present. Besides, as it can be seen in Fig. 7, the states that undergo a significant dependence with respect to the angle are also present when $\varepsilon=13$ and show the same electrical field distribution as the states previously

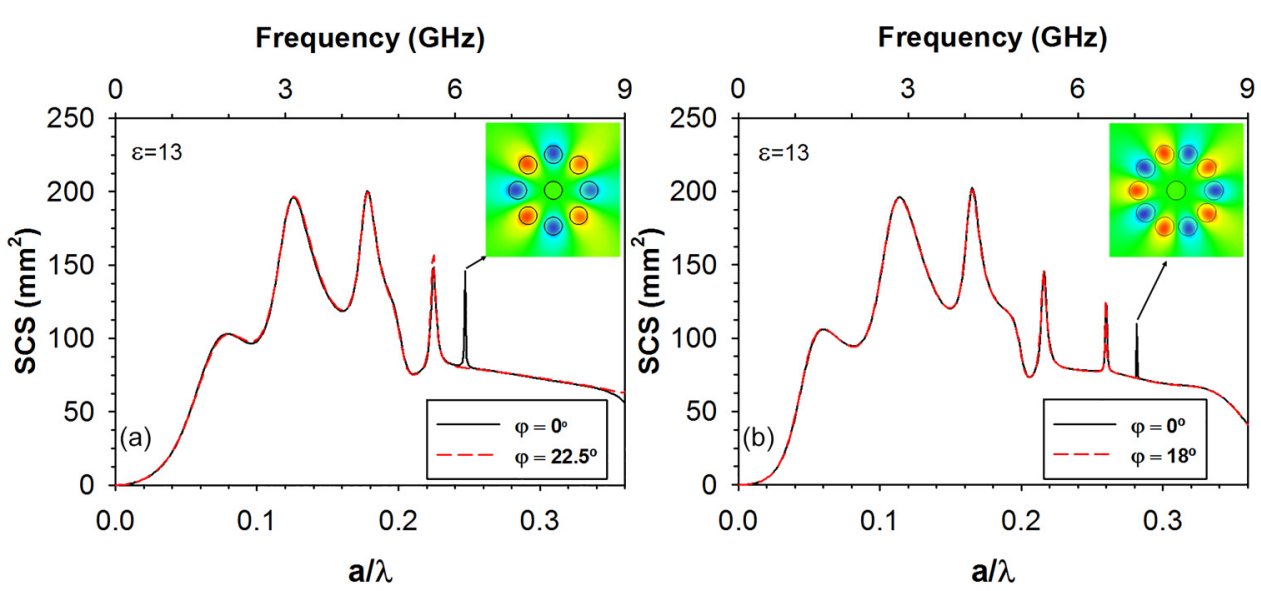

FIG. 7. Scattering Cross Section (SCS) as a function of the normalized frequency for the (a) octagonal and (b) decagonal $\mathrm{CR}$ with dielectric permittivity of the rods $\varepsilon=13$. The inset shows the electric field distribution of the states highly dependent on the incidence angle. 
analyzed with glass rods of permittivity equal to 4.5 . It is important to note that the electric field will be strongly localized inside the cylinders with a dielectric permittivity higher and could be employed to increase the sensitivity of the structure analyzed. Therefore, the selection of the material is not a strong limitation to the approach presented here for the use of $2 \mathrm{D}$ rings of cylinders as differential angle sensors.

\section{CONCLUSIONS}

In conclusion, we have studied collective resonators made with dielectric cylinder rings. We found that the same resonant states shown in bulk photonic quasicrystals are present in isolated octagons or decagons. Furthermore, we have found a particular state in each type of these resonator rings, whose excitation varies strongly with the angle of incident radiation. This state shows electric field intensity maxima inside each cylinder of the ring, which corresponds to individual Mie resonances with alternating polarities. This behavior is present regardless of the dielectric permittivity of the cylinders and in the two regular structures studied (decagonal and octagonal). The agreement between measurements and simulations is good which makes these structures excellent candidates to design differential angle sensors.

\section{ACKNOWLEDGMENTS}

This work has been supported by the Ministerio de Economía y Competitividad of Spain via Project No. TEC2014-51902-C2-2-R.

${ }^{1}$ E. Yablonovitch, "Inhibited spontaneous emission in solid-state physics and electronics," Phys. Rev. Lett. 58, 2059-2062 (1987).

${ }^{2}$ S. John, "Strong localization of photons in certain disordered dielectric superlattices," Phys. Rev. Lett. 58, 2486-2489 (1987).

${ }^{3}$ A. Della Villa et al., "Localized modes in photonic quasicrystals with Penrose-type lattice," Opt. Express 14, 10021 (2006).

${ }^{4} \mathrm{~K}$. Wang, "Light wave states in two-dimensional quasiperiodic media," Phys. Rev. B 73, 235122 (2006).

${ }^{5}$ S. Torquato and F. H. Stillinger, "Local density fluctuations, hyperuniformity, and order metrics," Phys. Rev. E. Stat. Nonlinear Soft Matter Phys. 68, 41113 (2003)

${ }^{6} \mathrm{~W}$. Man et al., "Photonic band gap in isotropic hyperuniform disordered solids with low dielectric contrast," Opt. Express 21, 19972-19981 (2013).

${ }^{7}$ M. Bayer et al., "Optical modes in photonic molecules," Phys. Rev. Lett. 81, 2582-2585 (1998).

${ }^{8}$ A. Armitage et al., "Polariton-induced optical asymmetry in semiconductor microcavities," Phys. Rev. B: Condens. Matter Mater. Phys. 58, 15367-15370 (1998).

${ }^{9}$ B. M. Möller, U. Woggon, M. V. Artemyev, and R. Wannemacher, "Photonic molecules doped with semiconductor nanocrystals," Phys. Rev. B 70, 115323 (2004).
${ }^{10}$ P. T. Rakich, M. A. Popović, M. Soljačić, and E. P. Ippen, "Trapping, corralling and spectral bonding of optical resonances through optically induced potentials," Nat. Photon. 1, 658-665 (2007).

${ }^{11}$ A. Nakagawa, S. Ishii, and T. Baba, "Photonic molecule laser composed of GaInAsP microdisks," Appl. Phys. Lett. 86, 041112 (2005).

${ }^{12} \mathrm{~S}$. Ishii and T. Baba, "Bistable lasing in twin microdisk photonic molecules," Appl. Phys. Lett. 87, 181102 (2005).

${ }^{13}$ K. A. Atlasov, K. F. Karlsson, A. Rudra, B. Dwir, and E. Kapon, "Wavelength and loss splitting in directly coupled photonic-crystal defect microcavities," Opt. Express 16, 16255 (2008).

${ }^{14}$ A. Yariv, Y. Xu, R. K. Lee, and A. Scherer, "Coupled-resonator optical waveguide: A proposal and analysis," Opt. Lett. 24, 711-713 (1999).

${ }^{15}$ Á Andueza, J. Pérez-Conde, and J. Sevilla, "Differential refractive index sensor based on photonic molecules and defect cavities," Opt. Express 24, 18807-18816 (2016).

${ }^{16} \mathrm{~K}$. Wang, "Light localization in photonic band gaps of quasiperiodic dielectric structures," Phys. Rev. B 82, 45119 (2010).

${ }^{17}$ E. I. Smotrova, A. I. Nosich, T. M. Benson, and P. Sewell, "Threshold reduction in a cyclic photonic molecule laser composed of identical microdisks with whispering-gallery modes," Opt. Lett. 31, 921-923 (2006).

${ }^{18}$ Y. W. Hu, Y. F. Xiao, Y. C. Liu, and Q. Gong, "Optomechanical sensing with on-chip microcavities," Front. Phys. 8, 475-490 (2013).

${ }^{19}$ M. J. Hartmann, F. G. S. L. Brandão, and M. B. Plenio, "Quantum manybody phenomena in coupled cavity arrays," Laser Photon. Rev. 2, 527-556 (2008)

${ }^{20}$ M. J. Hartmann, F. G. S. L. Brandão, and M. B. Plenio, "Effective spin systems in coupled microcavities," Phys. Rev. Lett. 99, 19-22 (2007).

${ }^{21} \mathrm{~S}$. V. Boriskina, "Spectrally engineered photonic molecules as optical sensors with enhanced sensitivity: A proposal and numerical analysis," J. Opt. Soc. Am. B 23, 1565-1573 (2006).

${ }^{22}$ F. Xia, M. Rooks, L. Sekaric, and Y. Vlasov, "Ultra-compact high order ring resonator filters using submicron silicon photonic wires for on-chip optical interconnects," Opt. Express 15, 11934-11941 (2007).

${ }^{23}$ J. V. Hryniewicz, P. P. Absil, B. E. Little, and R. A. Wilson, "Higher order filter response in coupled microring resonators," IEEE Photon. Technol. Lett. 12, 320-322 (2000).

${ }^{24}$ T.-W. Lu and P.-T. Lee, "Ultra-high sensitivity optical stress sensor based on double-layered photonic crystal microcavity," Opt. Express 17, 1518-1526 (2009).

${ }^{25}$ B. Z. Steinberg and A. Boag, "Splitting of microcavity degenerate modes in rotating photonic crystals-The miniature optical gyroscopes," J. Opt. Soc. Am. B 24, 142-151 (2007).

${ }^{26}$ C. Peng, Z. Li, and A. Xu, "Optical gyroscope based on a coupled resonator with the all-optical analogous property of electromagnetically induced transparency," Opt. Express 15, 3864-3875 (2007).

${ }^{27}$ J. Scheuer and A. Yariv, "Sagnac effect in coupled-resonator slow-light waveguide structures," Phys. Rev. Lett. 96, 1-4 (2006).

${ }^{28}$ C. Jin, B. Cheng, B. Man, Z. Li, and D. Zhang, "Two-dimensional dodecagonal and decagonal quasiperiodic photonic crystals in the microwave region," Phys. Rev. B 61, 10762-10767 (2000).

${ }^{29} \mathrm{~K}$. Wang, "Light wave states in two-dimensional quasiperiodic media," Phys. Rev. B: Condens. Matter Mater. Phys. 73, 235122 (2006).

${ }^{30}$ C. F. Bohren and D. R. Huffman, Absorption and Scattering of Light by Small Particles (Wiley, 1983).

${ }^{31}$ Á Andueza, K. Wang, J. Pérez-Conde, and J. Sevilla, "Optimal width of quasicrystalline slabs of dielectric cylinders to microwave radiation transmission contrast," J. Appl. Phys. 120, 083101 (2016).

${ }^{32} \mathrm{M}$. Clemens and T. Weil, "Discrete electromagnetism with the finite integration technique,” Prog. Electromagn. Res. 32, 65-87 (2001). 